\title{
Mechanical ventilation guidelines in lung lobectomy surgery and the quest to improve outcomes
}

\author{
Bilal Ansari ${ }^{1,2,3}$, Matteo Parotto ${ }^{1,2,3}$ \\ ${ }^{1}$ Department of Anesthesia, University of Toronto, Toronto, ON, Canada; ${ }^{2}$ Department of Anesthesia and Pain Management, Toronto General \\ Hospital, Toronto, ON, Canada; ${ }^{3}$ Interdepartmental Division of Critical Care Medicine, University of Toronto, Toronto, ON, Canada \\ Correspondence to: Matteo Parotto, MD, PhD. Department of Anesthesia and Pain Management, Toronto General Hospital, EN 442 - 200 Elizabeth \\ Street, M5G 2C4 Toronto, ON, Canada. Email: matteo.parotto@uhn.ca. \\ Comment on: Gao S, Zhang Z, Brunelli A, et al. The Society for Translational Medicine: clinical practice guidelines for mechanical ventilation \\ management for patients undergoing lobectomy. J Thorac Dis 2017;9:3246-54.
}

Submitted Nov 07, 2018. Accepted for publication Nov 09, 2018.

doi: $10.21037 /$ jtd.2018.11.52

View this article at: http://dx.doi.org/10.21037/jtd.2018.11.52

Mechanical ventilation is a life-saving treatment that changed patient care opportunities in perioperative and critical care medicine. Unfortunately, soon after its introduction into clinical practice it became evident how positive pressure ventilation held the potential to contribute to, and cause, lung injury-a phenomenon referred to as ventilator induced lung injury (VILI) (1).

The concept of lung-protective strategies arose from the need to mitigate VILI. Such strategies included low tidal volumes $(6-8 \mathrm{~mL} / \mathrm{kg}$ of predicted ideal body weight), limiting peak airway pressures and providing adequate positive end expiratory pressure (PEEP). The mortality benefits of such approaches were demonstrated initially in critically ill patients with lung injury. Subsequently, the benefits of protective approaches to mechanical ventilation were shown also in patients without prior lung injury undergoing elective abdominal and cardiac surgery $(2,3)$.

The perioperative care of individuals undergoing thoracic surgery poses unique challenges, given specific intraoperative ventilatory management needs and the high incidence of postoperative pulmonary complications (PPCs) (4).

One-lung ventilation (OLV), commonly used in thoracic surgery, poses added risks of hypoxemia and lung tissue oxidative stress injury (5), which can worsen morbidity. The requirements for delivering an adequate minute ventilation to one lung only may result in injurious volumes or pressures, and trauma to the lung deflated, operated on and subsequently re-inflated contributes to the risk of adverse events. However, data on the ideal perioperative ventilation management for these patients is scarce, and many questions remain unanswered to this date, including whether our knowledge of two-lung protective strategies can be extrapolated to OLV.

Recently in the Fournal of Thoracic Disease, Dr. Gao and colleagues from the Society for Translational Medicine have produced a set of clinical practice guidelines for mechanical ventilation in patients undergoing lung lobectomy surgery, by seeking the best evidence in the literature (6).

The authors produced a commendable effort to provide specific guidelines based on available data. They undertook a systematic literature search for studies investigating mechanical ventilation exclusively in patients undergoing lung lobectomy surgery published in the past 18 years, and narrowed their search from 461 citations down to 51 studies, using strict inclusion criteria. These papers were analyzed to identify the specific lung-protective strategies employed. Information such as mode of ventilation, inspiratory:expiratory (I:E) ratio, low tidal volumes and application of PEEP were examined in the context of OLV. In addition, they evaluated concepts such as therapeutic hypercapnia, pre- and post-operative non-invasive ventilation (NIV), open-lung strategies, low inspired oxygen concentration $\left(\mathrm{FiO}_{2}\right)$ and adjuvant drugs. An analysis of each intervention was provided along with a best-evidence recommendation.

The development of recommendations at present poses significant challenges, as the Authors highlight. As described above, a relatively small number of studies have evaluated 
the topic, and several limitations such as small sample sizes or lack of long term clinical outcomes evaluation prevent definitive conclusions.

Nonetheless, the Authors provide some important information for clinicians.

For example, Gao and colleagues report that using a PEEP of $5 \mathrm{cmH}_{2} \mathrm{O}$ and tidal volumes of $6-8 \mathrm{~mL} / \mathrm{kg}$ appear beneficial. It is important to notice that studies addressing this aspect include control groups that received $0 \mathrm{cmH}_{2} \mathrm{O}$ PEEP only. While this supports the concept that some PEEP improves outcomes (consistent with findings from other areas of anesthesia and critical care), it remains unknown what the optimal level of PEEP might be. Perhaps future studies will provide further guidance and help us understand whether an individualized PEEP titration may be more beneficial than a 'one size fits all' approach.

The Authors also support the use of hypercapnia in OLV. One of the by-products of a low tidal volume strategy is a rise in end-tidal carbon dioxide, and consequent hypercapnic acidosis (HCA). While this hypercarbia has traditionally been considered a passive phenomenon, it is increasingly clear that HCA is a potent biological agent. Of importance in the context of protective ventilation, HCA directly attenuates VILI $(7,8)$. These protective actions of HCA appear to be due in part to its anti-inflammatory effects $(9,10)$. As Gao and colleagues have reiterated, hypercapnia has been shown to be beneficial in a wide range of lung injury models, and their recommendation to utilize its anti-inflammatory qualities for OLV is sound. On the other hand, one should not "assume that hypercarbia is not harmful". Ample evidence exists to suggest that HCA acts as a double-edged sword. HCA impairs bacterial killing in the setting of prolonged lung infection (11), may delay healing following ARDS (12), and delays plasma membrane resealing following VILI, a key mechanism of repair following ARDS (13).

A more obvious and radical way of mitigating the risks of mechanical ventilation would be to avoid it altogether during surgery (14). Gao et al. explore this non-traditional approach to thoracic surgery by contemplating lobectomy in non-intubated patients as an alternative. Patients either receive intravenous sedation as an infusion which allows them to maintain spontaneous breathing, or receive epidural anesthesia or intercostal blocks. The surgical approach is thoracoscopic, in some cases uniportal, where the surgeon gives the patient a pneumothorax, allows the operative lung to collapse in order to perform the lobectomy, then inserts an intercostal drain at the end of the procedure and allows the lung to re-expand before completion of the procedure. This novel technique has potentially important benefits, but with the current data it remains too early to say if it can provide superior results compared with conventional methods.

Other recommendations from Dr. Gao et al. include the consideration for intraoperative alveolar recruitment, preferential use of pressure-controlled ventilation (PCV) or pressure-controlled volume-guaranteed ventilation (PCV-VG) over volume-controlled ventilation (VCV), post-operative continuous positive airway pressure (CPAP), application of the lowest $\mathrm{FiO}_{2}$ necessary to maintain satisfactory arterial oxygen saturation, I:E ratio of $1: 1$ or greater, perioperative use of adjuvant drugs such as nebulized budesonide, intravenous sivelestat and ulinastatin to attenuate the inflammatory response following OLV. Unfortunately, at present the data we have available to support such measures often arise from single studies of small sample size.

If we take a look at the literature on the management of thoracic surgery patients using broader search criteria, we notice some relevant studies. For instance, a number of recent studies have focused on the potential benefits of high-flow nasal oxygen (HFNO) therapy to reduce PPCs $(15,16)$. HFNO, as a therapy, provides a degree of CPAP, while guaranteeing adequate airways humidification and it may be better tolerated by patients in comparison to conventional NIV. The iPROVE-OLV study is an international randomized controlled trial (RCT) of lung-protective ventilation in OLV, and is currently underway. Early data from the iPROVE investigators show promise (17). The investigators advocate an open lung approach consisting of an alveolar recruitment maneuver followed by a PEEP adjusted to best respiratory system compliance, the results of which include lower driving pressure, improved lung compliance and a drop in PPCs. These results were mirrored by Rauseo and colleagues in a similar study of PEEP titration in patients undergoing lobectomy (18).

Dr. Gao and colleagues have made a valuable contribution by providing an evidence-based set of sensible guidelines for lung protection in patients undergoing lung lobectomy surgery. Their manuscript also reminds us of important unanswered questions in the field. This is an area of burgeoning interest to both thoracic surgeons and anesthesiologists, as clinicians and researchers strive to improve perioperative outcomes in this delicate population, who shows some of the highest rates of 
PPCs. Ongoing research is promising and may contribute important information in the near future. For what pertains the intraoperative care, the work by the iPROVE investigators will inform on the role of open lung approach, individualized PEEP titration, and reducing the driving pressure. With regards to the immediate postoperative period, we need guidance on strategies to prevent/reduce atelectasis, on the role of newer technology such as humidified HFNO therapy and/or NIV.

We hope that the next few years will hold refined approaches to guide the care of this patient population.

\section{Acknowledgements}

M Parotto is supported by an Early Investigator Award from the Department of Anesthesia, University of Toronto, Toronto, ON, Canada.

\section{Footnote}

Conflicts of Interest: The authors have no conflicts of interest to declare.

\section{References}

1. Tremblay LN, Slutsky AS. Ventilator-induced lung injury: from the bench to the bedside. Intensive Care Med 2006;32:24-33.

2. Futier E, Constantin JM, Paugam-Burtz C, et al. A trial of intraoperative low-tidal-volume ventilation in abdominal surgery. N Engl J Med 2013;369:428-37.

3. Costa Leme A, Hajjar LA, Volpe MS, et al. Effect of intensive vs moderate alveolar recruitment strategies added to lung-protective ventilation on postoperative pulmonary complications: a randomized clinical trial. JAMA 2017;317:1422-32.

4. Canet J, Gallart L, Gomar C, et al. Prediction of postoperative pulmonary complications in a populationbased surgical cohort. Anesthesiology 2010;113:1338-50.

5. Feng H, Wang GM, Qiao Y, et al. Effects of sevoflurane preconditioning on lung injury during one lung ventilation. Int J Clin Exp Med 2015;8:13634.

6. Gao S, Zhang Z, Brunelli A, et al. The Society for Translational Medicine: clinical practice guidelines for mechanical ventilation management for patients undergoing lobectomy. J Thorac Dis 2017;9:3246-54.

7. Laffey JG, Engelberts D, Duggan M, et al. Carbon dioxide attenuates pulmonary impairment resulting from hyperventilation. Crit Care Med 2003;31:2634-40.

8. Sinclair SE, Kregenow DA, Lamm WJ, et al. Hypercapnic acidosis is protective in an in vivo model of ventilatorinduced lung injury. Am J Respir Crit Care Med 2002; 166:403-8.

9. Laffey JG, Kavanagh BP. Carbon dioxide and the critically ill—too little of a good thing? Lancet 1999;354:1283-6.

10. Laffey JG, O'Croinin D, McLoughlin P, et al. Permissive hypercapnia-role in protective lung ventilatory strategies. Intensive Care Med 2004;30:347-56.

11. O'Croinin DF, Nichol AD, Hopkins N, et al. Sustained hypercapnic acidosis during pulmonary infection increases bacterial load and worsens lung injury. Crit Care Med 2008;36:2128-35.

12. O'Toole D, Hassett P, Contreras M, et al. Hypercapnic acidosis attenuates pulmonary epithelial wound repair by an NF- $\kappa B$ dependent mechanism. Thorax 2009;64:976-82.

13. Doerr CH, Gajic O, Berrios JC, et al. Hypercapnic acidosis impairs plasma membrane wound resealing in ventilator-injured lungs. Am J Respir Crit Care Med 2005;171:1371-7.

14. Del Sorbo L, Ranieri VM. We do not need mechanical ventilation any more. Crit Care Med 2010;38:S555-8.

15. Ansari BM, Hogan MP, Collier TJ, et al. A randomized controlled trial of high-flow nasal oxygen (Optiflow) as part of an enhanced recovery program after lung resection surgery. Ann Thorac Surg 2016;101:459-64.

16. Zochios V, Collier T, Blaudszun G, et al. The effect of high-flow nasal oxygen on hospital length of stay in cardiac surgical patients at high risk for respiratory complications: a randomised controlled trial. Anaesthesia 2018;73:1478-88.

17. iPROVE Network investigators, Belda J, Ferrando C, et al. The Effects of an Open-Lung Approach During One-Lung Ventilation on Postoperative Pulmonary Complications and Driving Pressure: A Descriptive, Multicenter National Study. J Cardiothorac Vasc Anesth 2018;32:2665-72.

18. Rauseo M, Mirabella L, Grasso S, et al. Peep titration based on the open lung approach during one lung ventilation in thoracic surgery: a physiological study. BMC Anesthesiol 2018;18:156.

Cite this article as: Ansari B, Parotto M. Mechanical ventilation guidelines in lung lobectomy surgery and the quest to improve outcomes. J Thorac Dis 2018;10(12):6396-6398. doi: $10.21037 /$ jtd.2018.11.52 